\title{
Optimization of MSA with Swift Particle Swarm Optimization
}

\author{
Amit Rathi \\ Banasthali University \\ Department of Electronics \\ Banasthali, Tonk, Rajasthan, India
}

\author{
Poonam Rathi \\ Genpact \\ Malviya nagar \\ Jaipur, Rajasthan, India
}

\author{
Ritu Vijay \\ Banasthali University \\ Department of Electronics \\ Banasthali, Tonk, Rajasthan, India
}

\begin{abstract}
In this Paper a new designing method is proposed for Circular Patch Micro strip Antenna (MSA) using an artificial search Algorithm named Particle swarm Optimization (PSO). It needs two stages for designing. Firstly circular patch MSA antenna needs modelling using some benchmark function. Then in second stage it's require inverse modelling using an artificial search algorithm (PSO) with some constraints. According to above steps first bandwidth of MSA is modelled using bench mark function as an input and resulted output are in form of frequency range, circular patch radius (r), ground plane length, substrate thickness, electrical thickness and dielectric loss tangent using Artificial search method. This paper presents the strategy that at the starting process cognition-learning random factor has more effect then social learning random factor. Gradually social learning random factor has more impact after learning cognition random factor to find out global best. The aim is to find out under above circumstances these modifications in PSO (Swift PSO) can give better result for optimization of Micro Strip Antenna (MSA).
\end{abstract}

\section{Keywords}

Artificial Search Algorithm, inverse modelling, Particle Swarm Optimization, Cognition Factor, Social Learning Factor, Local Search and Global Search, Micro Strip Antenna.

\section{INTRODUCTION}

Designing of MSA procedure, first modeling then inverse modeling is done. For this it require a system (Z), which have some input parameter like $S_{1}, S_{2}, S_{3} \ldots S_{n}$ for giving a desired output (Y) of the system (Figure 1). Y has its value in term of input parameter $\mathrm{S}_{1}, \mathrm{~S}_{2}, \mathrm{~S}_{3} \ldots \mathrm{S}_{\mathrm{n}}$. $\mathrm{Y}$ is modeled by the inverse modeling system $Z_{i}$ for getting desired output $Y_{d}$.

Main aim of this paper is designing of a circular patch MSA antenna after its modeling. After mathematical modeling of five input parameter frequency range, dimension width, ground plane length with substrate thickness, electrical thickness and dielectric loss tangent, it give output as a Bandwidth of MSA. After getting bandwidth then an artificial search algorithm is used as an inverse model to find bandwidth is as input for desire frequency range, dimension width, ground plane length with substrate thickness, electrical thickness and dielectric loss tangent as output, which is show in fig. 2

Here Particle swarm optimization (PSO) [1] [2] algorithm is used as a artificial search algorithm. PSO is to be efficient for any application optimization developed by Kennedy and Eberhart is based on the analogy of bird flocks or fish school movements.

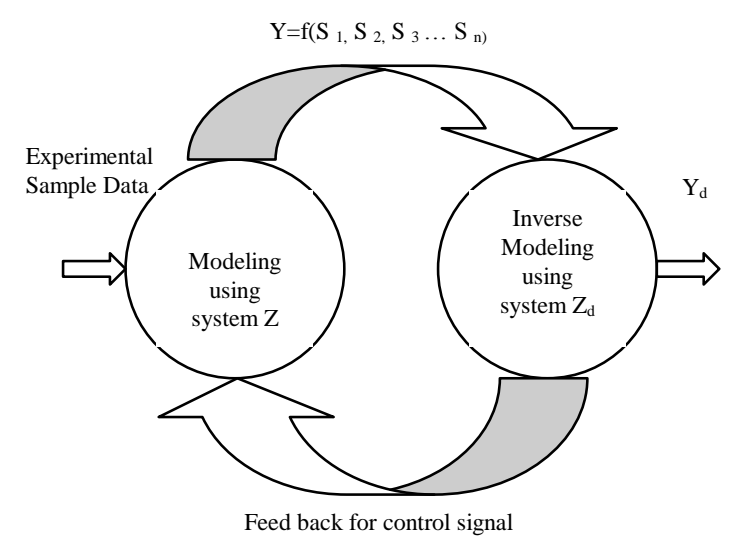

Fig. 1-Desired MSA designing steps using modeling and inverse modeling

The Cognition random factor and Social learning random Factor coefficient ( $\operatorname{rand}_{1}(\mathrm{)})$ and $\operatorname{rand}_{2}(\mathrm{)})$ are one of the parameter in Particle Swarm Optimization (PSO) [1]. This have important effect on balancing the local search and global search in Particle Swarm Optimization algorithm PSO, rand ${ }_{1}(\mathrm{)}$ is cognitive random coefficient has greater value then Social learning random Factor coefficient, rand ${ }_{2}()$ the particle goes to self learning .It controls the pull to the personal best position. $\mathrm{C}_{2}$ is the social rate

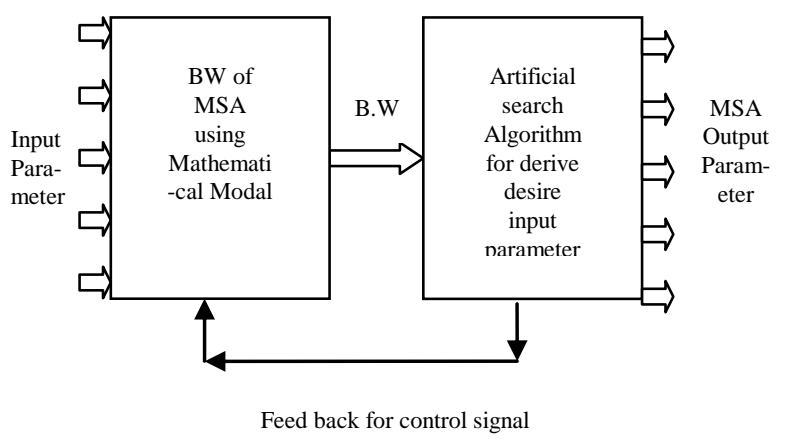

Fig.2- Searching of desired input Parameter for fixed BW

Coefficient .It controls the pull to the global best position. For any particulars it is necessary to know the things which are near to it. After getting the total knowledge of near things which is known as local best then it is better to take information further which is known as global best if it will not take local best position then it is difficult to take global best position. 
In this paper a new continuous time modal for Particle Swarm Optimization algorithm named Swift Particle Swarm Optimization (SPSO) is developed. In which rand ${ }_{1}\left(\right.$ ) and rand ${ }_{2}$ ( ) has varying value and the main goal of our work is investigation of the efficiency of new Particle Swarm Optimization algorithm (PSO) for MSA by taking rand ${ }_{1}()$ and rand ${ }_{2}()$ varying quantities for each iteration $(\mathrm{K})$.

A circular patch antenna with single coaxial feed is investigated. Aim of this paper best particle error, Physical thickness, operating frequency, electrical thickness, circular patch radius (r), dielectric loss tangent and bandwidth are investigated between circular patch antennas using standard PSO and circular patch antenna using modified PSO.

The remaining part of this paper is organized as follow. Section II briefly outlines the basics of the Particle Swarm Optimization algorithm (PSO) algorithms. Section III gives the information of modification of for Particle Swarm Optimization algorithm (PSO) in term of dynamics rand ${ }_{1}()$ and rand ${ }_{2}()$ for each iteration. In section IV introduction, scope and construction of circular patch MSA antenna is described. Experimental setting and results are described in section V and finally section VI described conclusion of this paper.

\section{STANDARD PSO ALGORITHM}

Particle Swarm Optimization algorithm is to be efficient for any application optimizations [1], [2], [3]. The Particle Swarm Optimization algorithm (PSO) searches for the global minimum of the cost-function, i.e., minimizes the cost-function of a problem by simulating movement and interaction of particles in a swarm. The position of a particle corresponds to one possible solution of the problem, i.e., it represents one point in the optimization space. Since we assume that there is no a priori knowledge of the optimization problem, there is equal possibility of choosing any point in the optimization space at the beginning of the optimization. Therefore, for Particle Swarm Optimization algorithm (PSO) starts with randomly chosen positions and velocities of particles. Each agent has information about the best position found by itself, $\mathrm{P}_{\text {best }}$ (which is the position vector in the optimization space), and the best position found by whole swarm, $\mathrm{G}$ best. The velocity vector for the calculation of the particle position in the next iteration is calculated as:

$\mathrm{V}_{n}=w^{*} \mathrm{~V}_{n-1}+\mathrm{C}_{1} *$ rand $\left(\mathrm{)} *\left(\mathrm{P}_{\text {best }}-\mathrm{X}_{\mathrm{n}-1}\right)+\mathrm{C}_{2}\right.$ rand ()$*\left(\mathrm{G}_{\text {best }}\right.$ $\left.-X_{n-1}\right)$

Where $\mathbf{V}_{n-1}$ is the particle velocity in the previous iteration, $w$ is inertia coefficient, rand ( ) is the function that generates uniformly distributed random numbers in the interval from 0.0 to $1.0, \mathrm{C}_{1}$ is the cognitive coefficient (it controls the pull to the personal best position), and $\mathrm{C}_{2}$ is the social-rate coefficient (it controls the pull to the global best position). The next position of the particle in the optimization space is calculated as:

$\mathrm{X}_{\mathrm{n}}=\mathrm{X}_{\mathrm{n}-1}+\mathrm{V}_{\mathrm{n}} \Delta \mathrm{t}$

Where $\Delta \mathbf{t}$ is most often considered to be of a unit value. It is found that particles might fly-out from the given optimization space if there are no limits for the velocity. Therefore, the maximal velocity $\mathrm{V}_{\text {max }}$ is introduced as another parameter of the for Particle Swarm Optimization algorithm (PSO). The maximal velocity, $\mathrm{V}_{\text {max }}$, represents the maximal percentage of the dynamic range of the optimization variable for which the velocity can change in successive movements of the particle.

\section{CONGNITION RANDOM FACTOR AND SOCIAL LEARNING RANDOM FACTOR FOR SWIFT PARTICLE SWARM OPTIMIZATION}

The velocity equation (1) of Particle Swarm Optimization algorithm (PSO) consists of three parts[1], [3]. In first part, w plays the role of balancing the global search and local search. Second and third parts contribute to the change of the velocity. The second part of the equation (1) is the "cognition" part, which represents the private thinking of the particle itself. The third part of the equation (1) is the "social part " which represents the collaboration among the particles. Without the first part of equation (1),all the particles will tend to move towards the same position. By adding the first parts, they have tendency to expand the search space that is they have ability to explore new area. So they have a global search capability by adding the first parts. In the second and third part of equation (1) it have two constant Cognition random factor rand ${ }_{1}(\mathrm{)})$ and Social learning random factor rand ${ }_{2}$ ( ) which also have very important role for particle to search their optimal value.

Proportional relationship of Cognition factor and Social Learning Factor for improving Particle Swarm Optimization (PSO) derived from standard Particle Swarm Optimization algorithm (PSO). The authors modified cognitive random factor (rand ${ }_{1}()$ ) and Social learning random factor (rand 2 ( )) for getting better efficiency. Which show that cognitive factor required first to search best position. After searching local best and knowing the information about local best then search for the global best.

In Proportional relationship of Cognition random factor (rand ${ }_{1}($ )) and Social Learning random Factor ( $\operatorname{rand}_{2}($ )) for improving Particle Swarm Optimization (PSO) is expressed as:

$$
\begin{aligned}
& \operatorname{rand}_{1}()=\left(1-\left(\mathrm{K}_{\mathrm{i}}-\mathrm{K}_{\min }\right) / \mathrm{K}_{\max }\right. \\
& \operatorname{rand}_{2}()=\mathrm{n}\left(\mathrm{K}_{\mathrm{i}}-\mathrm{K}_{\min }\right) / \mathrm{K}_{\max }
\end{aligned}
$$

Where-

$$
\begin{aligned}
& \operatorname{rand}_{1}()=\text { cognitive random factor } \\
& \operatorname{rand}_{2}()=\text { Social Learning random Factor } \\
& \mathrm{K}_{\mathrm{i}} \quad=\mathrm{i}^{\text {th }} \text { iteration } \\
& \mathrm{K}_{\text {min }} \quad=\text { predefined first iteration } \\
& \mathrm{K}_{\max } \quad \text { =last or final iteration } \\
& \mathrm{K}_{\mathrm{i}} \geq \mathrm{K}_{\max }
\end{aligned}
$$

Fig 3 shows the flow Chart of Enhanced Particle Swarm Optimization using Cognition factor and Social Learning Factor. 


\section{SCOPE AND STRUCTURE OF MICRO STRIP PATCH ANTENNA}

In telecommunication, there are several types of antennas, the most common of which is the micro strip patch antenna or patch antenna[4] [5].

Its name is attributed to the fact that it consists of a single metal patch suspended over a ground plane. The simplest patch antenna uses a patch, which is one half-wavelength-long with the dielectric loading included over a larger ground plane separated by a constant thickness. This type of configuration is showing in fig 4 , where $2 \mathrm{a}$ is the diameter of the circular patch antenna. Electrically large ground planes produce stable patterns and lower environmental sensitivity but of course make the antenna bigger. It isn't uncommon for the ground plane to be only modestly larger than the active patch. When a ground plane is close to the size of the radiator it can couple and produce currents along the edges of the ground plane, which also radiate.

The antenna pattern becomes the combination of the two sets of radiators [6] [10]. Wireless communications systems are in use in multitude of sizes ranging from small hand-held devices to devices mounted on vehicles. Modern wireless communication systems require wide bandwidth to provide high-speed data transmission. For optimum system performance, high radiation efficiency, small volume, simple and low-loss impedance matching to receive and transmit paths is prerequisites of the antennas [3]. Micro strip antennas have attracted much interest due to their small size, lightweight, low cost on mass production, low profile and easy integration with other components [7], [13]. The micro strip antenna is less suitable for modern communication system. These types of antenna are resonant at single frequency and it gives the poor gain as well as bandwidth [6], [7]. In recent year, much geometry is analyzed, in which rectangular, circular and triangular are mostly analyzed antenna geometries.

In this design, a circular patch antenna [8] [11] [12] [14] of proper dimension is simulated by IE3D software. A circular patch of $14.00 \mathrm{~mm}$ radius have permittivity $\varepsilon_{\mathrm{r}}=4.4$, substrate height $\mathrm{h}$ $=0.160 \mathrm{~cm}$, frequency range $=3 \mathrm{GHz}$, electrical thickness +0.24 , radius of circular patch $=14 \mathrm{~mm}$, dielectric loss tangent $=0.00194$ and bandwidth $(\%)=15$

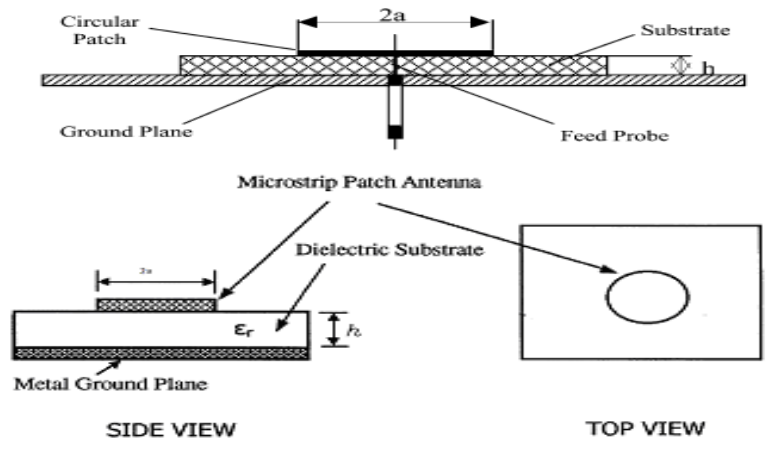

Fig. 4: Configuration of circular patch Antenna

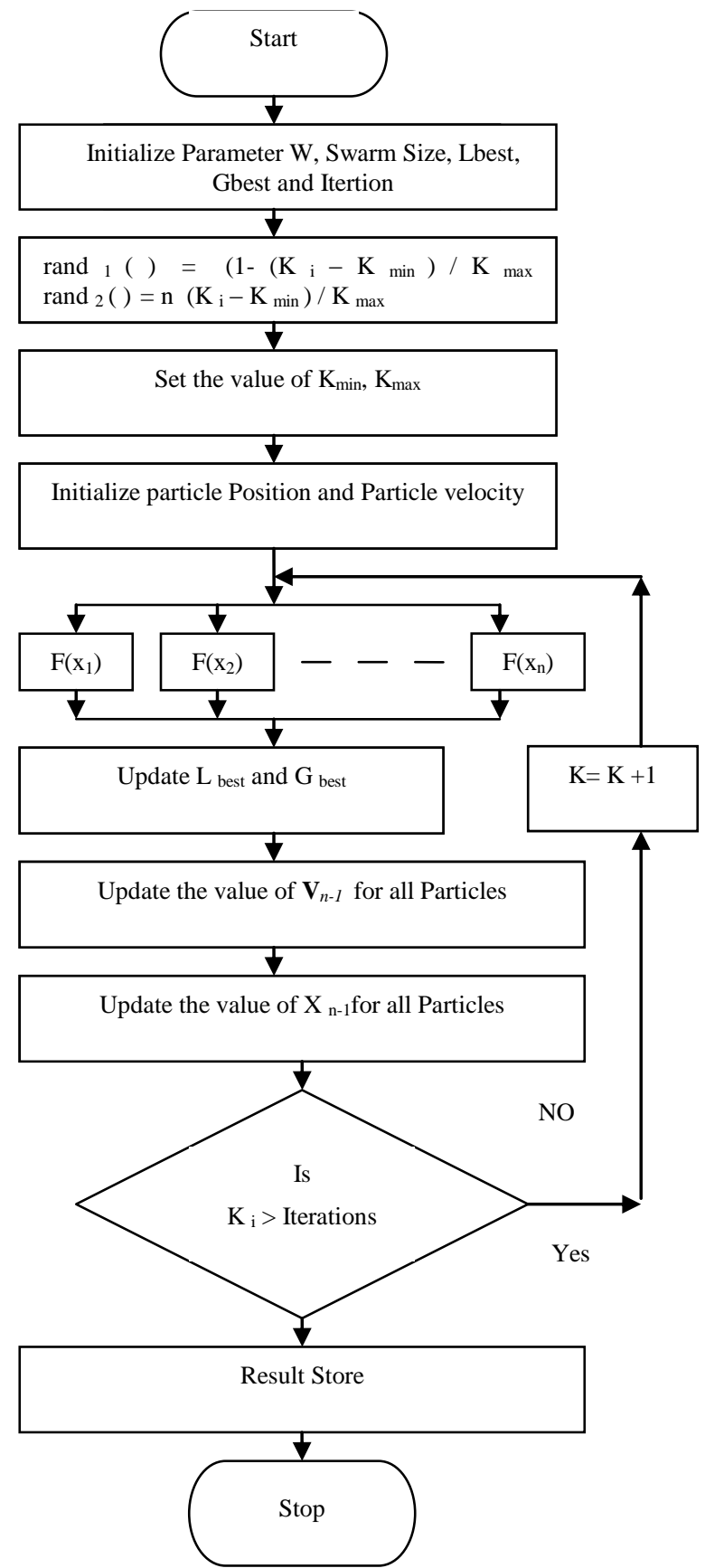

Fig 3: Flow Chart of Linear relationship of rand 1 ( ) and rand $_{2}$ ( ) for Swift Particle Swarm Optimization

\section{EXPERIMENT SETTING AND RESULT}

For experiment setting, test the new strategies to verify their performance. Using Benchmark functions to experiment: the swarm's size is 5 in all experiment, every function experiments 100 times independently, $\mathrm{w}=0.4$, rand ${ }_{1}\left(\right.$ ) and rand ${ }_{2}($ ) according to the equation. 
Benchmark functions are given as follows:

Rastrigin:

$$
f_{1}(x)=\sum_{i=1}^{n}\left[\left(x_{i}^{2}-10 \cos (2 \Pi x)+10\right]\right.
$$

Girewank:

$$
f_{2}(x)=\frac{1}{4000} \sum_{i=1}^{n}\left[\left(x_{i}^{2}-\prod_{i=1}^{n} \cos \left(\frac{x_{i}}{\sqrt{i}}\right)+1\right.\right.
$$

Schaffer:

$$
f_{3}(x)=0.5+\frac{\left(\sin \sqrt{\left(x_{1}^{2}+x_{2}{ }^{2}\right.}\right)^{2}-0.5}{\left[1+0.001\left(x_{1}{ }^{2}+x_{2}{ }^{2}\right)\right]^{2}}
$$

Benchmark functions' dimensions, the initial range, the criterion and the global optimum result is shown at Table 1Different parameter and their limitation range of MSA show in table 2.

Here Particle of PSO has five different dimensions so they search space region with different velocities. For fine searching, it is necessary to search with high velocity and terminated with low value.

Table 1. Benchmark functions

\begin{tabular}{|l|l|l|l|l|}
\hline Function & $\begin{array}{l}\text { Dime- } \\
\text { nsion }\end{array}$ & Initial range & Criterion & $\begin{array}{l}\text { The } \\
\text { global } \\
\text { optimu } \\
\mathrm{m} \\
\text { Result }\end{array}$ \\
\hline Rastrigin & 30 & $\begin{array}{l}{[-5.12,} \\
5.12]\end{array}$ & 50 & 0 \\
\hline Girewank & 30 & {$[-600,600]$} & 0.05 & 0 \\
\hline Schaffer f6 & 2 & {$[-100,100]$} & 0.00001 & 0 \\
\hline
\end{tabular}

Physical thickness (h), Frequency range (f $r$, Electrical thickness $\left(\mathrm{h} / \lambda_{\mathrm{d}}\right)$, circular patch radius $(\mathrm{r})$, Dielectric Loss Tangent $(\tan \delta)$ have varying value.

Table 2. Parameter and their limitation ranges of MSA

\begin{tabular}{|l|l|}
\hline MSA parameters & Range \\
\hline Frequency $\left(\mathrm{f}_{\mathrm{r}}\right)$ & {$[0.5,8.5]$} \\
\hline Physical Thickness $(\mathrm{h})$ & {$[0.7,20.12]$} \\
\hline Electrical thickness $\left(\mathrm{h} / \lambda_{\mathrm{d}}\right)$ & {$[0.0085,0.4248]$} \\
\hline circular patch radius $(\mathrm{r})$ & {$[8.76,21.64]$} \\
\hline Dielectric Loss Tangent $(\tan \delta)$ & {$[0.001,0.003]$} \\
\hline
\end{tabular}

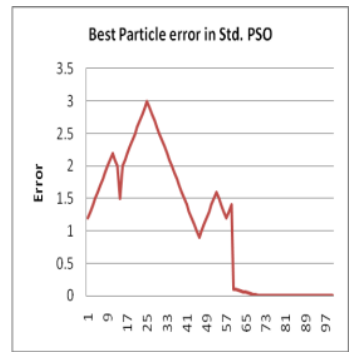

(a)

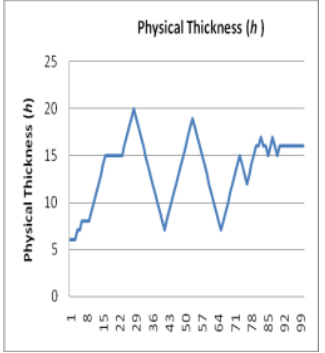

(b)
Maximum iteration is 100 in PSO and desired bandwidth is 15 . Figure 5 show each iteration errors of best particle and five variable obtained valves.

For evolution of performance, each iteration of PSO are performed by bench mark function for getting bandwidth and then this bandwidth is compared to desired bandwidth 15 . Above comparisons are shows by figure 6 .

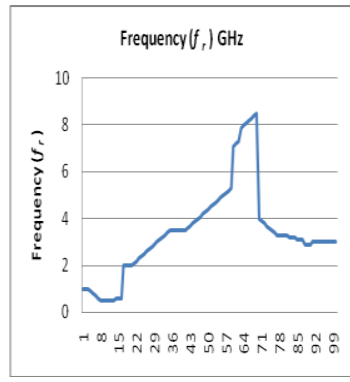

(c)

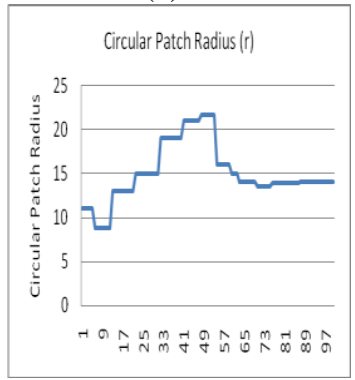

(e)

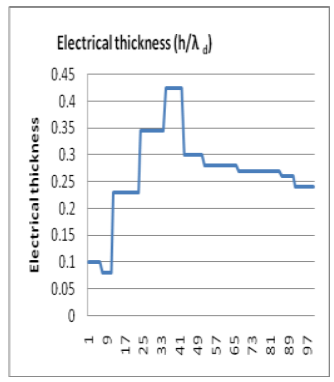

(d)

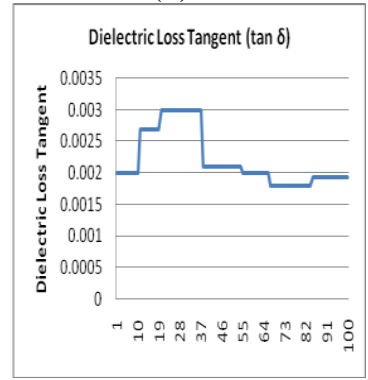

(f)
Fig. 5: (a) Error (b)Physical Thickness (c) Frequency (d) Electrical Thickness (e) Patch Radius (f) Dielectric Loss Tangent (g) Bandwidth obtained in Standard PSO

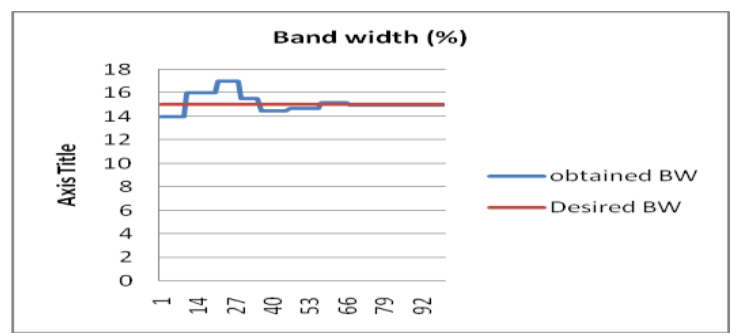

(g)

Fig. 6: Desired Bandwidth and obtained Bandwidth comparison

\subsection{Effect of modified PSO (SPSO)}

Applying modified PSO in above case figure 7 show each iteration error of the best Particle and obtained value of five variable. Table 3 shows different parameter of modified PSO algorithm. Table 4 shows the results of standard PSO and modified PSO. 
Table 3. Parameter of standard and Modified PSO

\begin{tabular}{|l|l|l|}
\hline $\begin{array}{l}\text { Algorithm } \rightarrow \\
\begin{array}{l}\text { Parameter } \\
\downarrow\end{array}\end{array}$ & Standard PSO & New Modified PSO \\
\hline w & 0.4 & 0.4 \\
\hline Swarm Size & 5 & 5 \\
\hline Iteration & 100 & 100 \\
\hline C1 & 1.4 & $\mathrm{n}\left(1-\left(\mathrm{K}_{\mathrm{i}}-\mathrm{K}_{\min }\right) / \mathrm{K}_{\max }\right.$ \\
\hline C2 & 1.4 & $\mathrm{n}\left(\mathrm{K}_{\mathrm{i}}-\mathrm{K}_{\min }\right) / \mathrm{K}_{\max }$ \\
\hline
\end{tabular}

Table 4. Results

\begin{tabular}{|l|l|l|}
\hline Parameter & $\begin{array}{l}\text { Iteration required } \\
\text { for standard PSO }\end{array}$ & $\begin{array}{l}\text { Iteration } \\
\text { required for } \\
\text { modify PSO }\end{array}$ \\
\hline Best Particle error & 60 & 45 \\
\hline Frequency $\left(\mathrm{f}_{\mathrm{r}}\right)$ & 92 & 80 \\
\hline Physical Thickness $(\mathrm{h})$ & 80 & 70 \\
\hline Electrical thickness $\left(\mathrm{h} / \boldsymbol{\lambda}_{\mathrm{d}}\right)$ & 94 & 82 \\
\hline circular patch radius $(\mathrm{r})$ & 88 & 72 \\
\hline Dielectric Loss Tangent $(\tan \delta)$ & 86 & 71 \\
\hline Band width $(\%)$ & 68 & 42 \\
\hline
\end{tabular}

For evolution of performance, each iteration of SPSO are performed by bench mark function for getting bandwidth and then this bandwidth is compared to desired bandwidth 15 . Above comparison is show by figure 8 .

\section{CONCLUSION}

A Particle Swarm Optimization (PSO) based approach is proposed for designing microstrip antenna using some benchmark function.

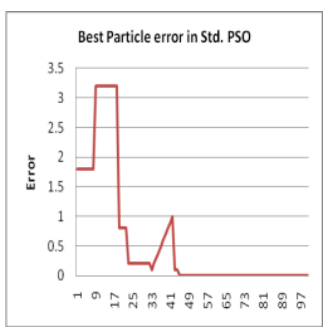

(a)

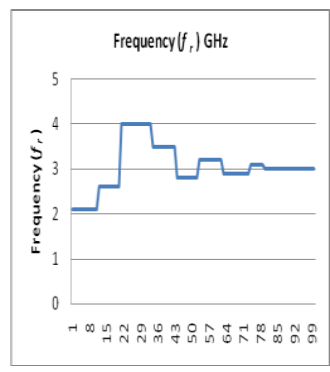

(c)

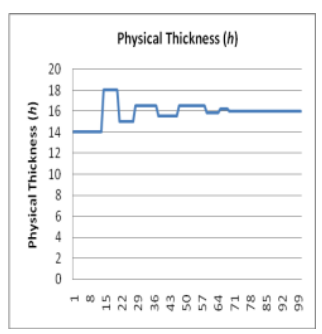

(b)

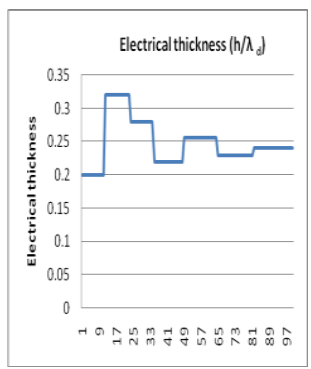

(d)

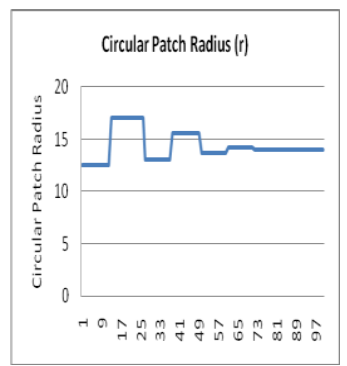

(e)

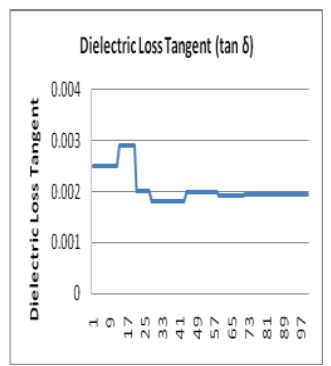

( f )
Fig. 7: (a) Error (b)Physical Thickness (c) Frequency (d) Electrical Thickness (e) Patch Radius (f) Dielectric Loss Tangent (g) Bandwidth obtained in Modified PSO

This give better result and required less computational time by maintaining good quality of solution as compared to original Particle Swarm Optimization algorithm (PSO). The author have proposed to take first cognitive random factor large as compared to social random factor then for next step gradually decreased rand $_{1}(\mathrm{)})$ and increased rand $\mathrm{C}_{2}(\mathrm{)}$ give better result as compare to existing algorithm. The proposed method is new and computationally efficient and accurate which show in table 3

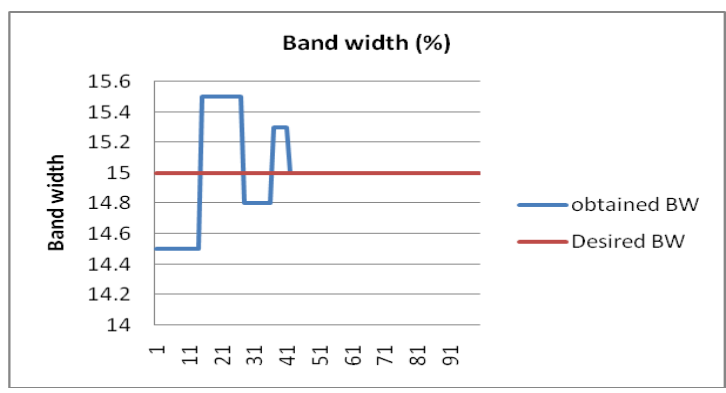

( g )

Fig. 8: Desired Bandwidth and Optimized Bandwidth comparison in Modified PSO

\section{REFERENCES}

[1] J. Kennedy and R. C. Eberhart, 1995, Particle swarm optimization, in Proc. IEEE Int. Conf. Neural Networks, pp. 1942-1948 .

[2] K. M. Bakwad, S.S. Patnayak, B.S. Sohi, Swapna Devi, Sastri V.R.S. Gollapudi, Ch. Vidya Sagar, P.K.Patra, 2008, Small population Based Modified Parallel Particle swarm Optimization for Motion Estimation, in Proc. IEEE Int.

[3] J. J. Liang, A. K. Qin, P. N. Suganthan, and S. Baskar , 2006, Comprehensive learning particle swarm optimizer for global optimization of multimodal functions," IEEE Trans. Evol. Comput., vol. 10, no. 3, pp. 281-296, Jun. 2006.

[4] Hadi Sadoghi Yazdi, Mehri Sadoghi Yazdi, 2009, Particle swarm optimization -Based Rectangular Microstrip Antenna Designing, International Journal of Computer and Electrical Engineering, Vol. 1, No. 4, October, 2009 1793-8163.

[5] Kara M, 1996, A simple technique for the calculation of bandwidth of rectangular microstrip antenna elements with 
various substratethicknesse, Microw Opt Technol Lett 12:16- 20 .

[6] Pozar and Schaubert,1992, Microstrip Antennas, Proceedings of the IEEE, vol. 80.

[7] Kara M, 1996, A novel technique to calculate the bandwidth of rectangular microstrip antenna elements with thick substrates, Microw Opt Technol Lett 12:59-64.

[8] Sagiroglu S, Guney K, Erler M, 1999, Calculation of bandwidth for electrically thin and thick rectangular microstrip antennas with the use of multilayered perceptrons, Int J Microw Comput Aided Eng 9:277-286.

[9] Kaplan A, Guney K, Ozer S,2001, Fuzzy associative memories for the computation of the bandwidth of rectangular microstrip antennas with thin and thick substrates, Int J Electron 88:189-195.
[10] Bahl IJ, Bhartia P, 1980, Microstrip antennas, Artech House, Canton, MA.

[11] Pozar DM, 1983, Considerations for millimeter wave printed antennas, IEEE Trans Antennas Propagat 31:740- 747.

[12] Sang-Hyuk Wi, Yong-Shik Lee, and Jon Gwan Yook, 2007, Wideband Microstrip Patch Antenna With U Shaped Parasitic Elements, IEEE Transaction On Antenna and Propagation, Vol. 55, No.4, April 2007.

[13] J. J. Liang, and P. N. Suganthan, 2005, Dynamic MultiSwarm Particle Swarm Optimizer, IEEE Swarm Intelligence Symposium, pp. 124-129, Pasadena, CA, USA.

[14] J. J. Liang, A. K. Qin, P. N. Suganthan and S. Baskar, 2006, Comprehensive Learning Particle Swarm Optimizer for Global Optimization of Multimodal Functions, IEEE Trans. on Evolutionary Computation, Vol. 10, No. 3, pp. 281-295. 